Supporting Information for Langmuir manuscript LA - 2007 - 02099t:

\title{
Is Latex Surface Charge an Important Parameter for Foam Stabilization?
}

Sarah L. Kettlewell, Andreas Schmid, Syuji Fujii,\$ Damien Dupin and Steven P. Armes*

Department of Chemistry, Dainton Building, University of Sheffield, Brook Hill, Sheffield, South Yorkshire, S3 7HF, UK.

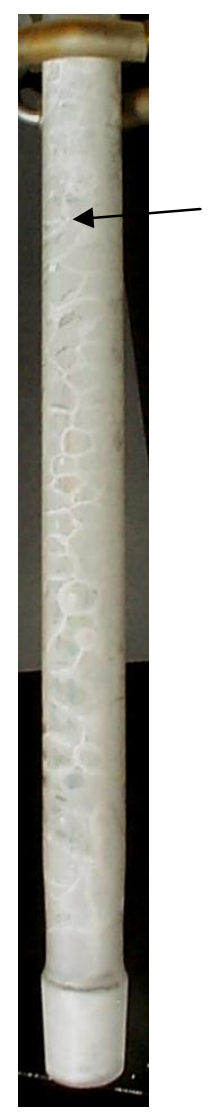

\section{Foam produced using a foam column}

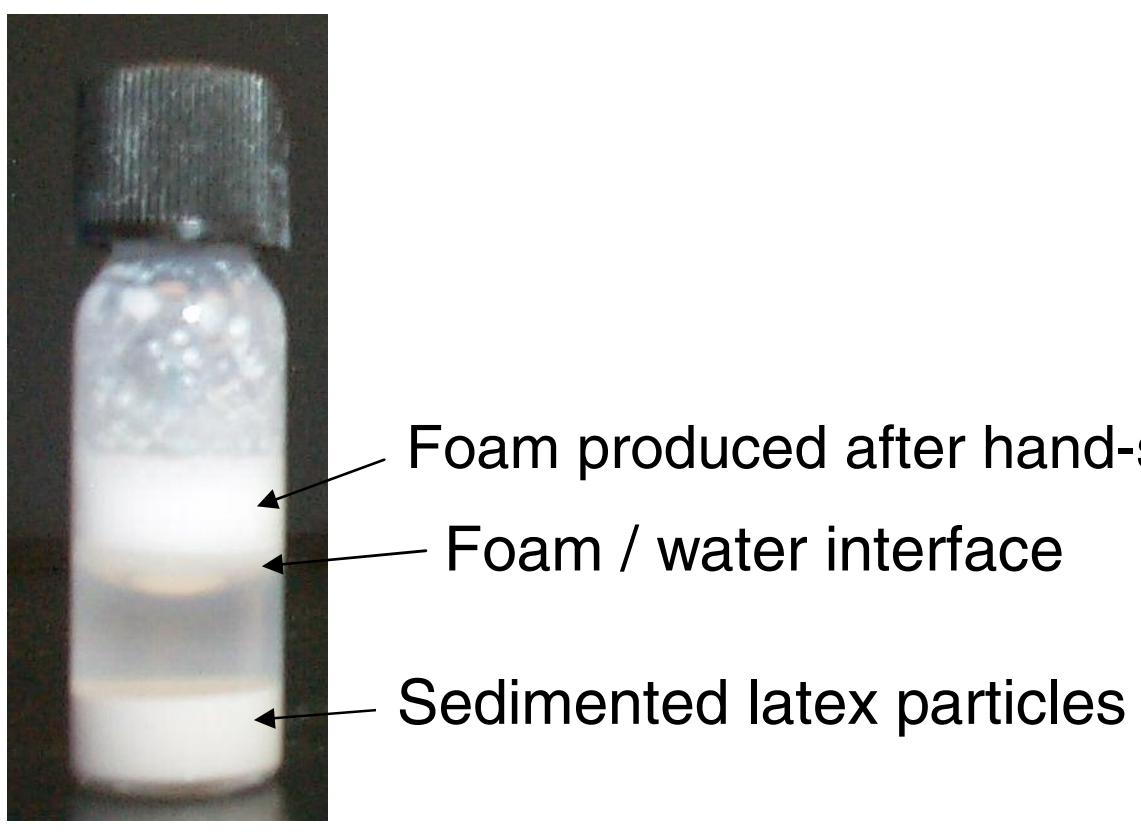

Figure 1S. Digital photographs of the long-lived foams generated from a $10.0 \mathrm{wt} . \%$ aqueous dispersion of an PEGMA-AIBA-PS latex using either a foam column or by hand-shaking. In the right-hand image, the sample was allowed to stand for two weeks, which allows the relatively large latex particles to sediment under gravity and hence reveal more clearly the foam volume above the meniscus. 


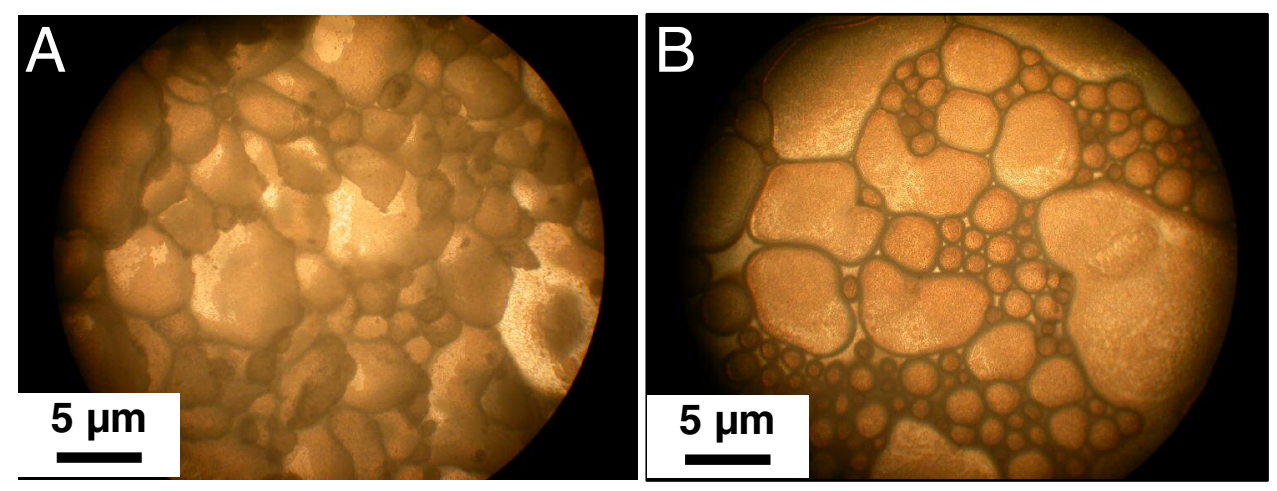

Figure 2S. Optical micrographs of wet foam bubbles stabilized (A) by the $724 \pm 81 \mathrm{~nm}$ chargestabilized cationic AIBA-PS latex particles and (B) by the $800 \pm 138 \mathrm{~nm}$ sterically-stabilized cationic PEGMA-AIBA-PS latex particles.

\section{${ }^{1}$ H NMR Characterisation of Latex Composition}

As shown overleaf, the four equivalent aliphatic ethylene glycol (EG) protons due to the PEGMA chains are clearly visible at $\delta 3.7$ for the PEGMA-AIBA-PS latex dissolved in $\mathrm{CD}_{2} \mathrm{Cl}_{2}$ (see Figure $3 \mathrm{~S}$, spectrum 'B') but not for the AIBA-PS latex (Figure 3S, spectrum 'A'), as expected. The integral of this PEGMA peak can be compared with the aromatic peak integral due to the styrene residues at $\delta 6.3-7.3$. Thus the molar ratio of EG to styrene units can be calculated, which in turn gives a PEGMA mass content for the PEGMA-AIBA-PS latex of 1.16 wt.\%. From this value, the surface absorbed amount, $\Gamma$, of the PEGMA stabiliser was estimated to be around $1.6 \mathrm{mg} \mathrm{m}^{-2}$ using the equation As $=3 / \mathrm{p} . \mathrm{R}$, where As is the specific surface area, $p$ is the latex density and $\mathrm{R}$ is the mean particle radius. In this calculation, it is assumed that all the PEGMA stabiliser is located at the surface of the polystyrene particles, which is highly likely given the post-grafting method used to attach the stabiliser. 


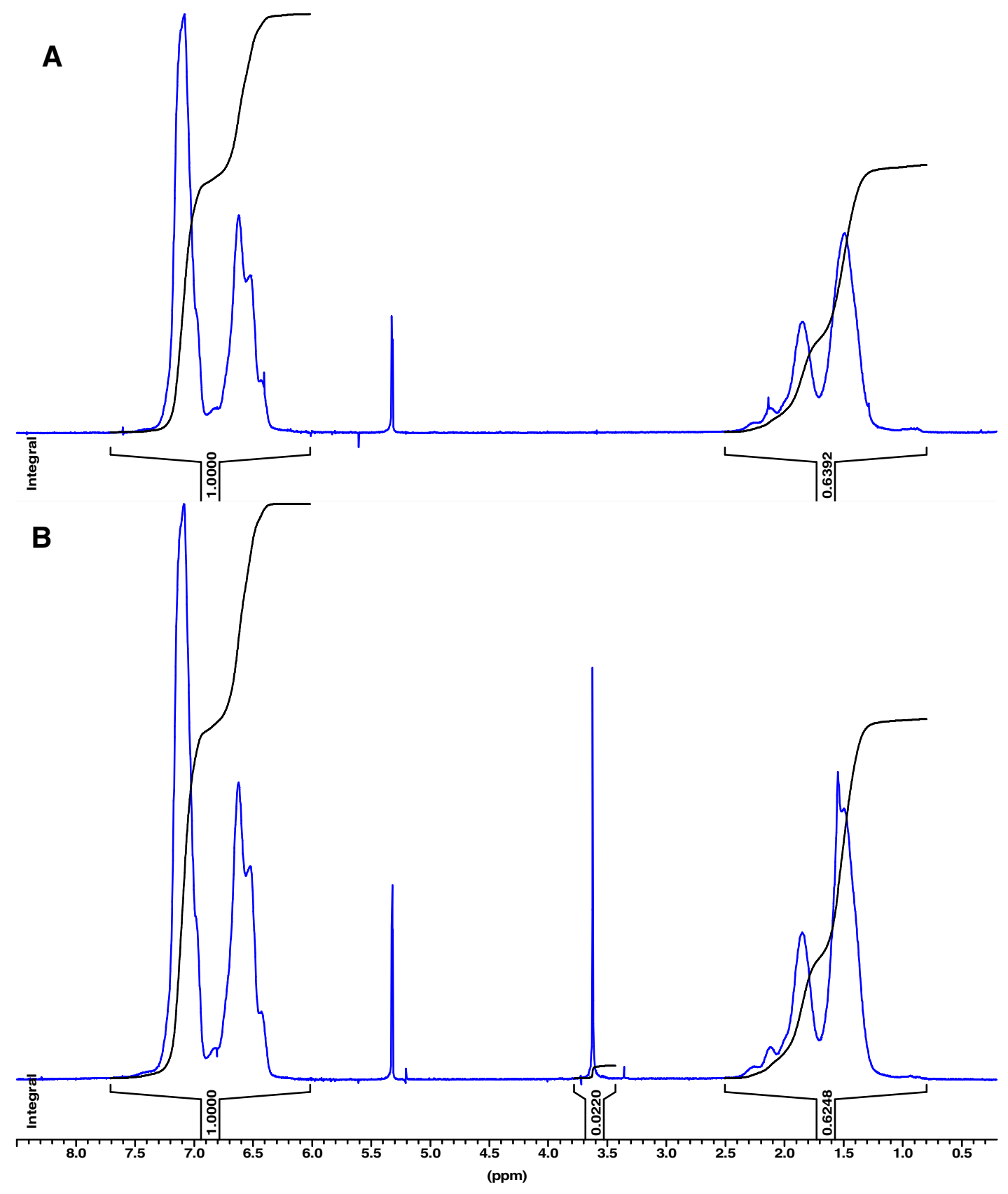

Figure 3S. ${ }^{1} \mathrm{H}$ NMR spectra recorded in $\mathrm{CD}_{2} \mathrm{Cl}_{2}$ for (A) the AIBA-PS latex and (B) the PEGMA-AIBAPS latex that confirm the presence of the PEGMA stabilizer in the PEGMA-AIBA-PS latex. 

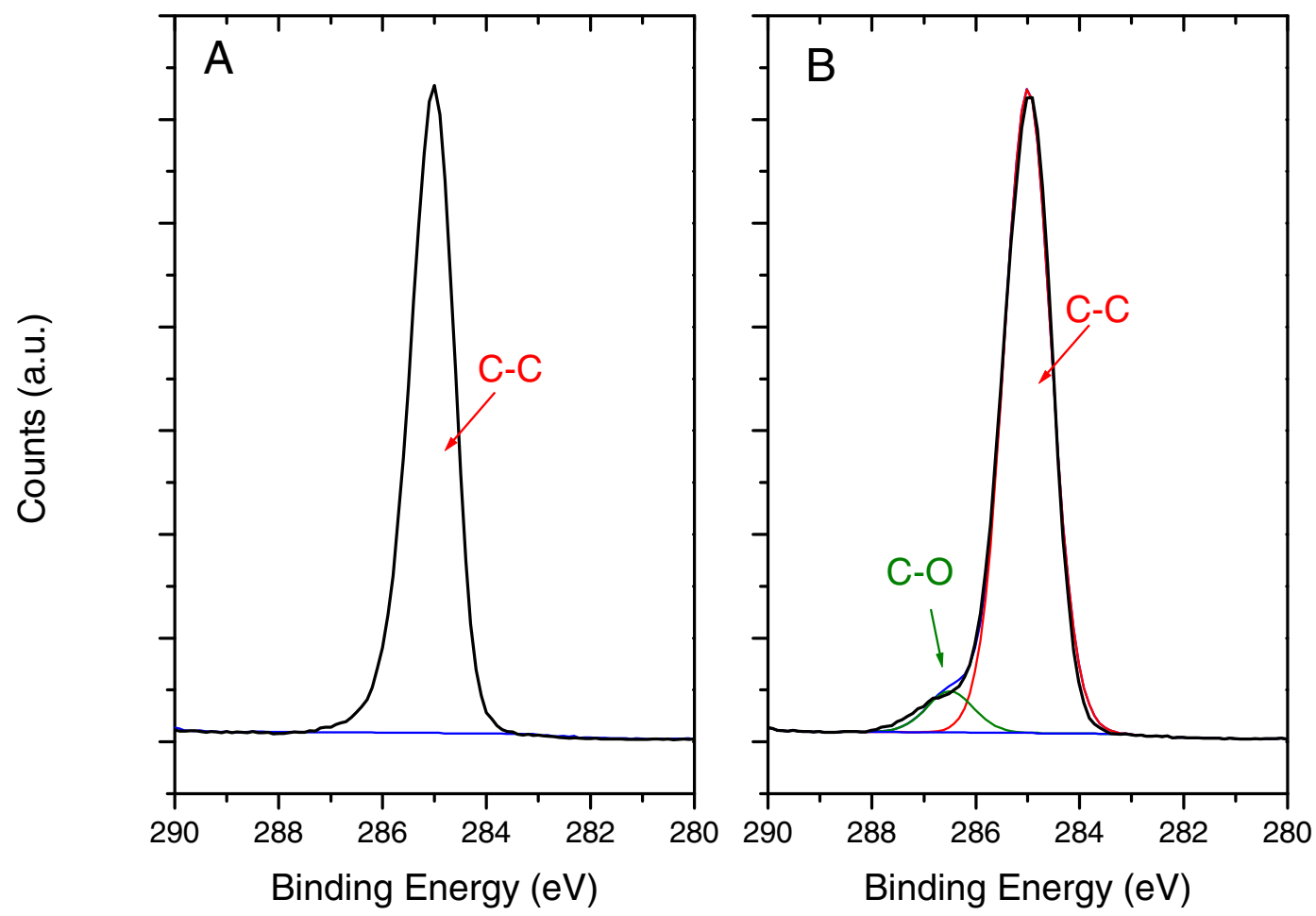

Figure 4S. Core-line C1s x-ray photoelectron spectra recorded for (A) the AIBA-PS latex and (B) the PEGMA-AIBA-PS latex. The C-O feature at approximately $286.5 \mathrm{eV}$ is only present in the PEGMAAIBA-PS sample and is attributed to the PEGMA component. Thus XPS confirms that the PEGMA stabilizer is indeed present on the latex surface. 